\title{
Numerical investigation of domain walls in constrained geometries
}

\author{
Hans Fangohr, Jürgen P Zimmermann, and Richard P Boardman \\ School of Engineering Sciences, University of Southampton \\ Southampton, SO17 1BJ, United Kingdom \\ David C Gonzalez, Cornelis H de Groot \\ School of Electronics and Computer Science \\ University of Southampton
}

(Dated: December 6, 2008)

\begin{abstract}
In recent years, magnetic domain wall structures in ferromagnetic nanowires have attracted growing attention, opening paths to develop novel devices which exploit magnetoresistive effects. A reduction of the domain wall length in geometrically constrained areas has been predicted and observed. In this article, we consider a rectangular constriction (width $s_{0}$, length $2 d_{0}$ ) in form of a thin film, attached to a rectangular pad (width $s_{1}$ ) on either side. The material considered is $\mathrm{Ni}\left(M_{\mathrm{s}}=490 \mathrm{kA} / \mathrm{m}\right)$ with a weak in-plane anisotropy $\left(K_{1}=2000 \mathrm{~J} / \mathrm{m}^{3}\right)$. We investigate the dependence of the domain wall length as a function of the constriction geometry. Micromagnetic simulations are used to systematically study the head-to-head domain walls between head-to-head domains (case A) and Néel walls between sidewise domain orientations (case B). We present the resulting domain wall length $w$ as a function of $2 d_{0}$ and $s_{0}$ and analyze the magnetization patterns. A reduction of the domain wall length to below $11 \mathrm{~nm}$ is found (where the corresponding unconstrained domain wall length is $69 \mathrm{~nm}$ ). For constriction lengths above a critical value (case B only), the single $180^{\circ}$ domain wall splits into two $90^{\circ}$ domain walls.
\end{abstract}

PACS numbers: $75.40 . \mathrm{Mg}, 75.70 . \mathrm{Ak}, 75.60 . \mathrm{Ch}, 75.75 .+\mathrm{a}, 07.05 . \mathrm{Tp}$

\section{INTRODUCTION}

Bruno [1] predicted that the width of domain walls in ferromagnetic systems can be reduced by the geometry of the system. In particular, he has shown that the presence of a geometric constriction (see for example figure 1) can make it energetically favorable for the domain wall to reduce its length.

Subsequent work includes experimental verification of this prediction [2] and numerical micromagnetic studies to further the understanding of these compressed domain walls. In Ref. [3] the domain wall configuration and the domain wall resistance in constrictions of the Bruno geometry model I have been studied numerically. (Model II and III [1] are other geometric realization of constrictions). A numerical and experimental study [4] has investigated Néel domain walls (case B). In Ref. [5] the equilibrium domain wall configurations for an out-of-plane anisotropy in geometry constrictions of model I have been simulated. In Ref. [6] and [7] the micromagnetism of constrictions which taper (linearly) towards the thinnest connection and then widen again have been studied. In this work, we study the domain wall length systematically as a function of width and length of the Bruno model I.

Section II introduces the definition of the domain wall length used in this work. Section III describes the geometry, the parameters we have varied and the material parameters. We present our results and discussion in section IV, before we close with a summary in section $\mathrm{V}$.

\section{DOMAIN WALL LENGTH}

Bruno[1] has provided the following definition of the domain wall length $w$

$$
w=4\left[\int_{-\infty}^{\infty}(\dot{\phi}(x))^{2} \mathrm{~d} x\right]^{-1}
$$

where $\phi(x)$ is the rotation angle of the magnetization, and $\dot{\phi}(x)=\frac{\mathrm{d} \phi}{\mathrm{d} x}$ for a domain wall in $x$-direction. The prefactor is chosen such that for a domain wall in an unconstrained geometry and in the absence of demagnetization effects, $w=2 \sqrt{A / K}$ where $A$ is the exchange coupling strength and $K$ the uniaxial anisotropy strength.

We will use Bruno's definition (1) to compute the domain wall length $w$ in the constrained geometries we investigate here.

\section{SIMULATION PARAMETERS}

The thin film geometry we investigate here is defined by Bruno [1] as constriction model I, and shown in figure 1. Two pads of height $s_{1}=40 \mathrm{~nm}$ and width $p_{\mathrm{x}}=50 \mathrm{~nm}$ are connected by a less wide connection of length $2 d_{0}$ and width $s_{0}$. The magnetization is generally fixed at the left and the right ends of the geometry in opposite directions. In particular, we study two configurations: (A) the magnetization is pinned in the $+x$ direction at the left side and in the $-x$ direction at the right side, and $(\mathrm{B})$ the magnetization is pinned in the $+y$ direction 


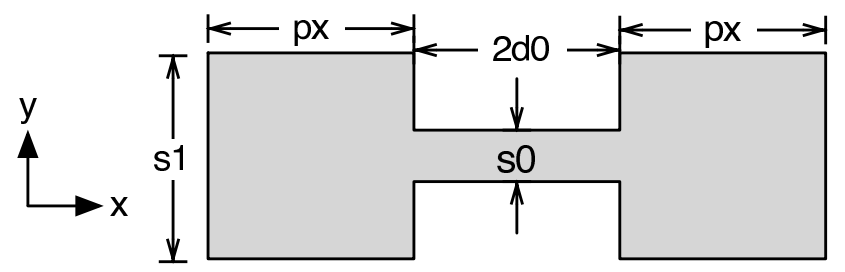

FIG. 1: The geometry under investigation (proposed by [1] as "model I"). Panel width $s_{1}=40 \mathrm{~nm}$ and panel length $p_{\mathrm{x}}=$ $50 \mathrm{~nm}$ are constant in this study, $s_{0}$ and $d_{0}$ vary. For case A, we have an uniaxial anisotropy in $x$-direction, for case B an uniaxial anisotropy in $y$-direction.

at the left side and in the $-y$ direction at the right side. In this study, we vary the constriction width $s_{0}$ to take values $2,6,10,14,18,22,26,30,34$ and $38 \mathrm{~nm}$, and vary the constriction length $2 d_{0}$ to take values $2,52,102,152$, 202, 252, $302 \mathrm{~nm}$. This spans a configuration space of 70 different geometries for cases A and B, each.

The material we investigate is Nickel which can be tailored to have a weak uniaxial anisotropy by applying a magnetic field during the growth process. We use the material parameters (following [8], p17): saturation magnetization $M_{s}=490 \mathrm{kA} / \mathrm{m}$, exchange coupling $A=$ $9 \cdot 10^{-12} \mathrm{~J} / \mathrm{m}$, and uniaxial anisotropy $K_{1}=2000 \mathrm{~J} / \mathrm{m}^{3}$. We use the finite difference simulation tool OOMMF [9] with a simulation cell size of $1 \mathrm{~nm}^{3}$. We study the thinfilm limit and set the thickness of the film in $z$-direction to be $1 \mathrm{~nm}$.

The simulation of a large $\left(2 d_{0}=900 \mathrm{~nm}\right)$ system without a constriction (i.e. $s_{1} / s_{0}=1$ ) and without demagnetization effects, results in a domain wall length $w=134.1651 \mathrm{~nm}$ for both case A and B, computed using equation (1). This is in agreement with the analytical result $\delta_{B 2}=2 \sqrt{A / K}=134.1641 \mathrm{~nm}$. Taking the demagnetization field into account, the domain wall length becomes $w=w_{\mathrm{A}} \equiv 69 \mathrm{~nm}$ for case $\mathrm{A}$. For case $\mathrm{B}$, the domain wall splits into two parts (see section IV B), and we obtain $w=w_{\mathrm{B}}=98 \mathrm{~nm}$ (for the joint length of the $90^{\circ}$ domain walls). In the next section, we systematically study the influence of the demagnetization field and geometry which both affect the domain wall length. We use $w_{\mathrm{A}}=69 \mathrm{~nm}$ and $w_{\mathrm{B}}=98 \mathrm{~nm}$ to normalize the length of the domain walls in the presentation of the results.

\section{RESULTS}

\section{A. Anisotropy in $x$-direction (case A)}

We pin the magnetization in $+x$ and $-x$ direction at either side of the geometry and vary the width and height of the constriction as described in section III. For each configuration we compute the equilibrium magnetization configuration using OOMMF, and use (1) to find the domain wall length $w$. We obtain $\phi(x)$ using $\phi(x)=\arccos \left(\bar{M}_{\mathrm{x}}(x) / M_{\mathrm{s}}\right)$ where $\bar{M}_{\mathrm{x}}$ is the $x$ -
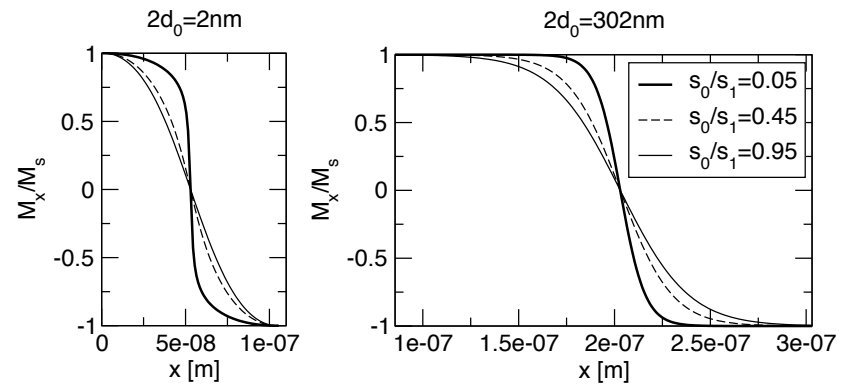

FIG. 2: Case A: Plot of the normalized $M_{\mathrm{x}}$ component for three different values of $s_{0}\left(s_{1}=40 \mathrm{~nm}\right)$ at $2 d_{0}=2 \mathrm{~nm}$ (left) and at $2 d_{0}=302 \mathrm{~nm}$ (right).

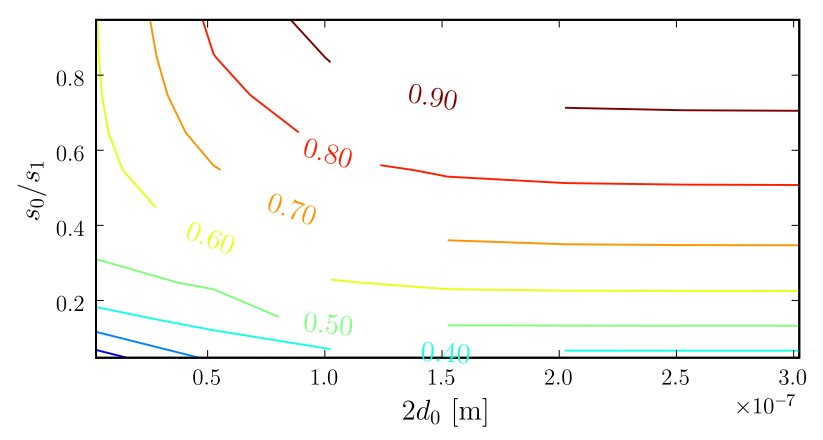

FIG. 3: Case A: Plot of the normalized domain wall length $w / w_{\mathrm{A}}$ as a function of $2 d_{0}$ and $s_{0} / s_{1}$.

component of the magnetization, averaged over $y$ and $z$, i.e. $\bar{M}_{\mathrm{x}}(x)=\frac{1}{l_{\mathrm{y}}} \frac{1}{l_{\mathrm{z}}} \int_{-0.5 l_{\mathrm{y}}}^{0.5 l_{\mathrm{y}}} \int_{-0.5 l_{\mathrm{z}}}^{0.5 l_{\mathrm{z}}} M_{\mathrm{x}}(x, y, z) \mathrm{d} z \mathrm{~d} y$. The domain wall length $w$ is insensitive to the choice of $l_{\mathrm{y}}$ and $l_{\mathrm{z}}$ : we obtain the same $w$ (to within less than $1 \%$ ) if we use $l_{\mathrm{y}}=s_{0}$ or $l_{\mathrm{y}}=1 \mathrm{~nm}$.

Figure 2 (right) shows $M_{\mathrm{x}}$ for $2 d_{0}=302 \mathrm{~nm}$ and three different values of $s_{0}$. It is clearly visible how the domain wall width reduces with decreasing ratio $s_{0} / s_{1}$. The reduced domain wall lengths $w / w_{\mathrm{A}}$ are 0.37 for $s_{0} / s_{1}=$ $0.05,0.77$ for $s_{0} / s_{1}=0.45$ and 1.00 for $s_{0} / s_{1}=0.95$.

Figure 2 (left) shows $M_{\mathrm{x}}$ for $2 d_{0}=2 \mathrm{~nm}$ and three different values of $s_{0}$. The reduced domain wall lengths $w / w_{\mathrm{A}}$ are 0.16 for $s_{0} / s_{1}=0.05,0.55$ for $s_{0} / s_{1}=0.45$ and 0.60 for $s_{0} / s_{1}=0.95$.

A systematic representation of the reduced domain wall length for all geometries simulated is given in figure 3 . For the smallest geometry $\left(2 d_{0}=2 \mathrm{~nm}, s_{0} / s_{1}=0.05\right)$, we find $w=0.16 w_{A}$ (i.e. $\left.w=10.8 \mathrm{~nm}\right)$.

\section{B. Anisotropy in $y$-direction (case B)}

For configuration case $\mathrm{B}$, we pin the magnetization to point to the $+y$ and $-y$ direction at the left and right border of the pads, respectively, and use an uniaxial anisotropy in the $y$-direction. Figure 4 shows $\bar{M}_{\mathrm{y}}(x)$ (i.e. 

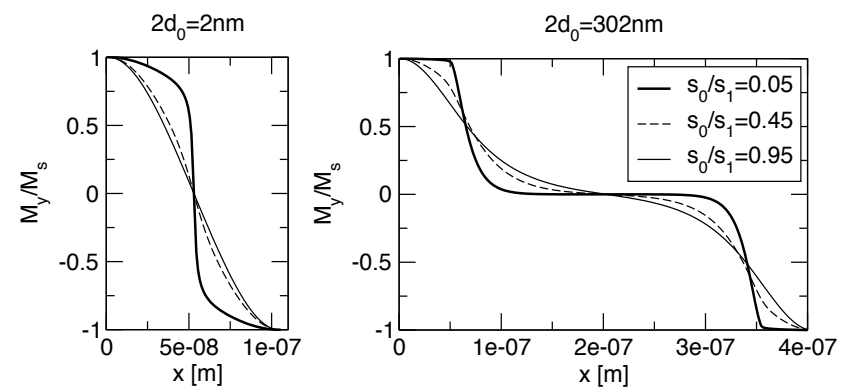

FIG. 4: Case B: Plot of the normalized $M_{\mathrm{y}}$ component for three different values of $s_{0}\left(s_{1}=40 \mathrm{~nm}\right)$ at $2 d_{0}=2 \mathrm{~nm}$ (left) and at $2 d_{0}=302 \mathrm{~nm}$ (right).

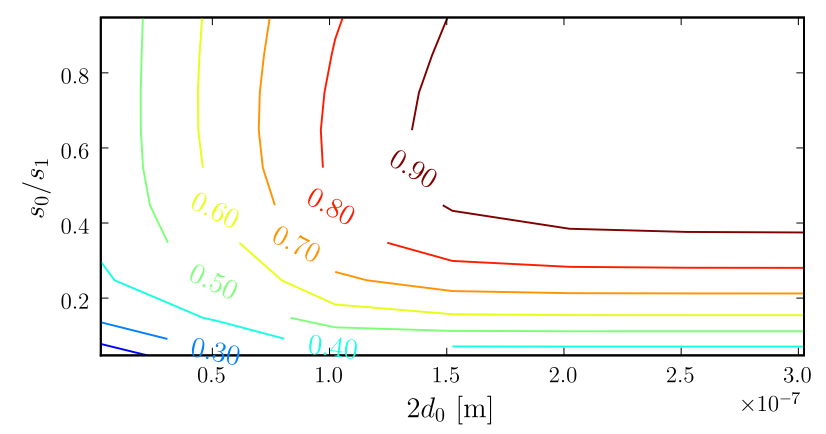

FIG. 5: Case B: Plot of the normalized domain wall length $w / w_{\mathrm{B}}$ as a function of $2 d_{0}$ and $s_{0} / s_{1}$. Where the domain wall splits into two $90^{\circ}$ domain walls in the constriction, the value for $w$ is the sum of the lengths of both $90^{\circ}$ walls.

the average of $M_{\mathrm{y}}$ over $y$ and $z$, corresponding to $\bar{M}_{\mathrm{x}}(x)$ in section IV A) for the same geometries as in figure 2.

For $2 d_{0}=302 \mathrm{~nm}$ (Fig 4 right), all three curves show a feature that is most clearly visible for $s_{0} / s_{1}=0.05$ : the $180^{\circ}$ rotation of the magnetization splits into two $90^{\circ}$ domain walls, in agreement with [4]. This reduces the demagnetization energy associated with the surface charges in the constriction. This domain wall splitting is not observed in case A due to the parallel alignment of constriction and easy axis. The $90^{\circ}$ domain walls are located just within the constriction area. Note that the definition (1) cannot distinguish between two $90^{\circ}$ domain walls or one $180^{\circ}$ domain walls. The values for $w / w_{\mathrm{B}}$ are 0.34 for $s_{0} / s_{1}=0.05,0.95$ for $s_{0} / s_{1}=0.45$ and 1.00 for $s_{0} / s_{1}=0.95$.

For $2 d_{0}=2 \mathrm{~nm}$ (Fig 4 left) the domain walls are qualitatively similar to case $\mathrm{A}$. The values for $w / w_{\mathrm{B}}$ are 0.15 for $s_{0} / s_{1}=0.05,0.42$ for $s_{0} / s_{1}=0.45$ and 0.43 for $s_{0} / s_{1}=0.95$.

Figure 5 shows a summary of the reduced domain wall length for case B (as figure 3 shows for case A). The transition from one $180^{\circ}$ domain wall to two $90^{\circ}$ domain walls happens for all ratios $s_{0} / s_{1}$ that we have studied between $2 d_{0}=2 \mathrm{~nm}$ and $2 d_{0}=52 \mathrm{~nm}$ (this is consistent with [4]). The smallest observed domain wall at $s_{0} / s_{1}=$ 0.05 and $2 d_{0}=1 \mathrm{~nm}$ is $14 \mathrm{~nm}$.

\section{SUMMARY}

We have carried out micromagnetic calculations to study the compression of domain walls in constrictive geometries. We find that choosing a suitable geometry, the domain wall length can be reduced by more than a factor of 6 in comparison to the unconstricted domain wall length (in the presence of demagnetization effects). Both the ratio $s_{0} / s_{1}$ as well as the length $d_{0}$ of the constriction are important parameters. A domain wall splitting into two $90^{\circ}$ walls for large values of $d_{0}$ is not observed for head-on-head (case A) domains.

\section{Acknowledgments}

We acknowledge funding from EPSRC (GR/T09156/01) for this work. We thank the anonymous reviewers for their helpful contributions to this manuscript.
[1] P. Bruno, Phys. Rev. Lett. 83, 2425 (1999).

[2] O. Pietzsch, A. Kubetzka, M. Bode, and R. Wiesendanger, Phys. Rev. Lett. 84, 5212 (2000).

[3] J. D. Burton, A. Kashyap, M. Y. Zhuravlev, R. Skomski, a. E. Y. Tsymbal, S. S. Jaswal, O. N. Mryasov, and R. W. Chantrell, Appl. Phys. Lett. 85, 251 (2004).

[4] P. Jubert, R. Allenspach, and A. Bischof, Phys. Rev. B 69, 220410(R) (2004).

[5] P.-O. Jubert and R. Allenspach, Journal of Magnetism and Magnetic Materials 290-291, 758 (2005).
[6] G. Li, Y. Zhai, P. Wong, D. Niu, Y. Lu, S. Lepadatu, and Y. Xu, IEEE Transactions on Magnetics 43, 2830 (2007).

[7] G. Sarau, S. Gliga, R. Hertel, , and C. M. Schneider, IEEE Transactions on Magnetics (2007).

[8] H. Kronmüller and M. Fähnle, Micromagnetism and the Microstructure of Ferromagnetic Solids (Cambridge University Press, 2003), 1st ed.

[9] M. Donahue and D. Porter, Object Oriented MicroMagnetic Framework (OOMMF), www.nist.gov/oommf. 